\title{
Pulmonary Arteriovenous Fistula Associated with Unequal Branching of the Pulmonary Artery System
}

\author{
Satoshi Morimoto, Yukihisa Fujita, Yoshinobu NaKagaki, Yoshinobu Iwasaki, \\ Taizo NaKamura, Shuji KatoH* and Masao NAKAGawA
}

\begin{abstract}
A 60-year-old woman was admitted to our hospital with shortness of breath and was diagnosed to have pulmonary arteriovenous fistula with unequal branching of the pulmonary artery system, as assessed by pulmonary angiography. The concomitant occurrence has not been reported previously. Although the etiology of these abnormalities is unknown, these abnormalities are considered to have occurred during an early stage of fetal development. The analysis of a concomitant occurrence of these abnormalities may be useful in elucidating the etiology of pulmonary arteriovenous fistula.
\end{abstract}

(Internal Medicine 34: 1019-1022, 1995)

Key words: pulmonary angiography, embolization therapy, Rendu-Osler-Weber disease, hereditary hemorrhagic telangiectasia

\section{Introduction}

Since the report by Churton in 1897 (1), more than 450 cases of pulmonary arteriovenous fistula have been reported (2). This abnormality has frequently been associated with hereditary hemorrhagic telangiectasia (Rendu-Osler-Weber disease) (2). Most pulmonary arteriovenous fistulas are congenital while some are acquired. We report a case of congenital pulmonary arteriovenous fistula associated with unequal branching of the pulmonary artery system (2).

\section{Case Report}

A 60-year-old woman with a 2-year history of productive cough and a 10-year history of shortness of breath was admitted to our hospital in June 1991, complaining of progressive shortness of breath. Family history was unremarkable. Physical examination revealed normal heart sounds and no pathologic heart murmurs. Coarse crackles were audible bilaterally in the lower lung fields. Cyanosis was noted on the extremities, but no clubbing of the fingers was seen. No thoracic bruit was audible. No telangiectasia was observed on the skin or mucosa.

Laboratory tests revealed a hemoglobin of $13.5 \mathrm{~g} / \mathrm{dl}$ and a hematocrit of $41.6 \%$. Results of liver function tests were normal.
The blood gas values were as follows: $\mathrm{PaO}_{2}, 69.3 \mathrm{mmHg}$ and $\mathrm{PaCO}_{2}, 51.0 \mathrm{mmHg}$, with a $\mathrm{pH}$ of 7.382 and a bicarbonate of $29.8 \mathrm{mEq} / \mathrm{l}$ in room air. Pulmonary function tests indicated a vital capacity (VC) of $1,160 \mathrm{cc}, \% \mathrm{VC} 48 \%$, forced expiratory volume (FEV) $1.0610 \mathrm{cc}$, FEV 1.0\% (G) 51\%, and residual volume $1,830 \mathrm{cc}$. Electrocardiogram and echocardiogram were normal. A chest X-ray film showed a normal heart size (cardiothoracic ratio 47\%), a reticular shadow in bilateral lower lung fields, and a well-delineated, homogeneous, $3-\mathrm{cm}$ long nodule that seemed to continue from a pulmonary artery in the right middle lung field (Fig. 1). Tomography of the right lung revealed that the superior part of the nodule was comprised of a pulmonary artery, and the inferior part was comprised of a pulmonary vein. Computed tomography scans demonstrated that the superior and anterior portion of the nodule was comprised of a pulmonary artery, and the inferior and posterior portion was comprised of a pulmonary vein. Bronchiectatic change was also seen in bilateral lower lobes.

A pulmonary scintigraphy after intravenous injection of technetium-99m-labeled macroaggregated albumin revealed decreased tracer uptake in the right superior and inferior lung fields and the left inferior lung fields. Pulmonary inhalation scintigraphy could not be performed because the patient had difficulty in inhaling the trace gas. Bronchoscopy showed normal branching of the bronchial system. Pulmonary angio-

From the Second Department of Medicine, Kyoto Prefectural University of Medicine, Kyoto and *the Department of Medicine, Murakami Memorial Hospital, Asahi University, Gifu

Received for publication January 6, 1995; Accepted for publication July 5, 1995

Reprint requests should be addressed to Dr. Satoshi Morimoto, the Second Department of Medicine, Kyoto Prefectural University of Medicine, 465 Kawaramachi Hirokoji, Kamigyo-ku, Kyoto 602 


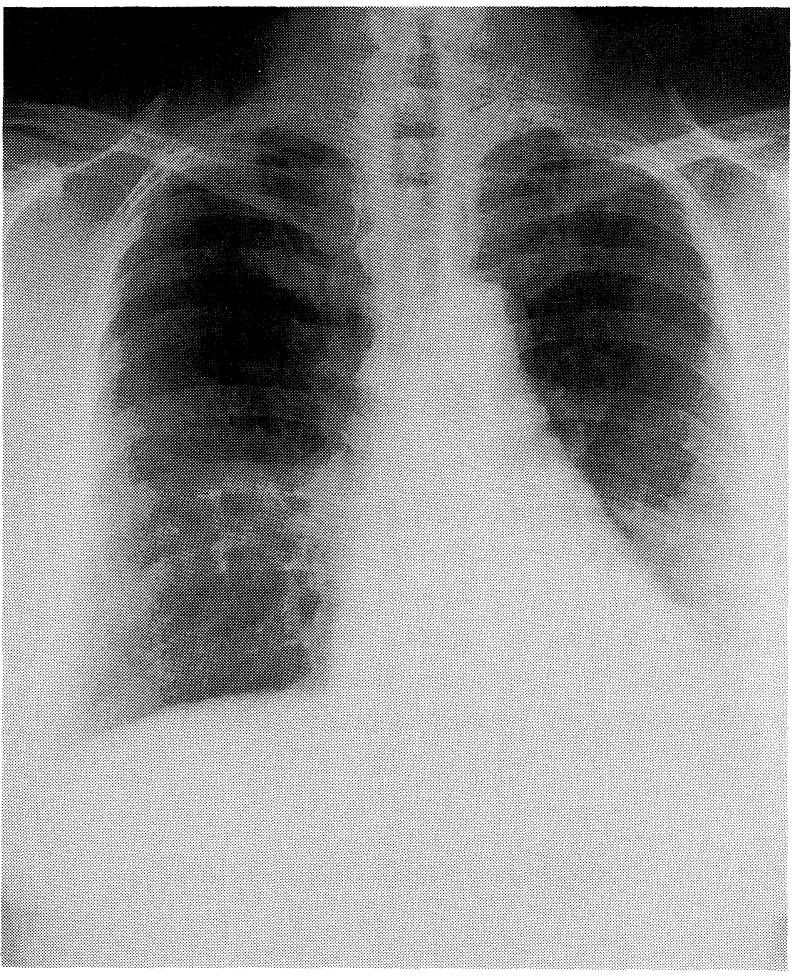

Figure 1. A chest X-ray (posteroanterior view). A welldelineated and homogeneous nodule that seems to continue from a pulmonary artery in the right middle lung field is observed.

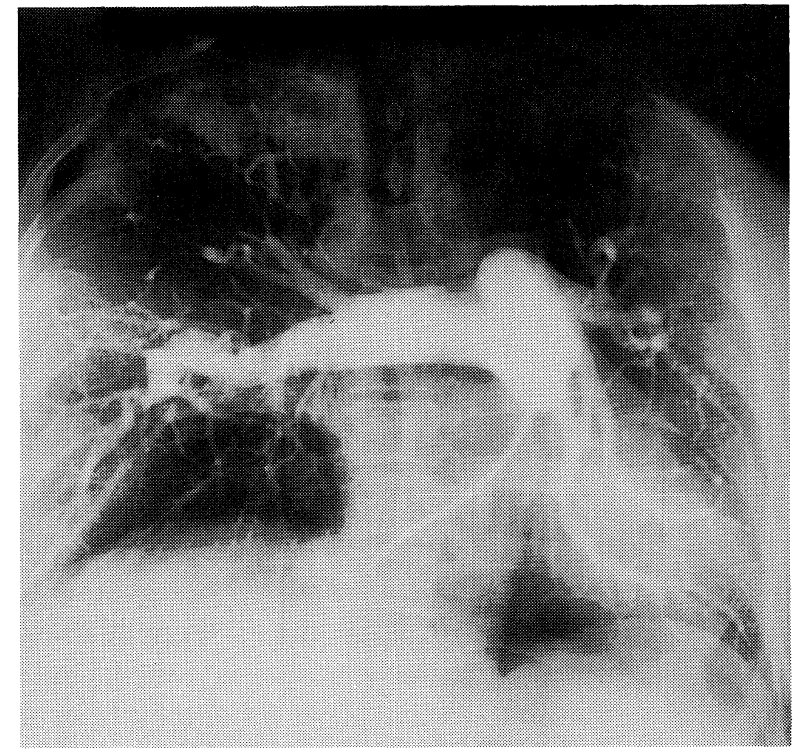

Figure 2. Pulmonary artery angiogram (posteroanterior view). Branches of the pulmonary artery to the bilateral inferior lobes are narrow and branch abnormally. The right superior, the right inferior, and the left superior lobes are hypovascular.
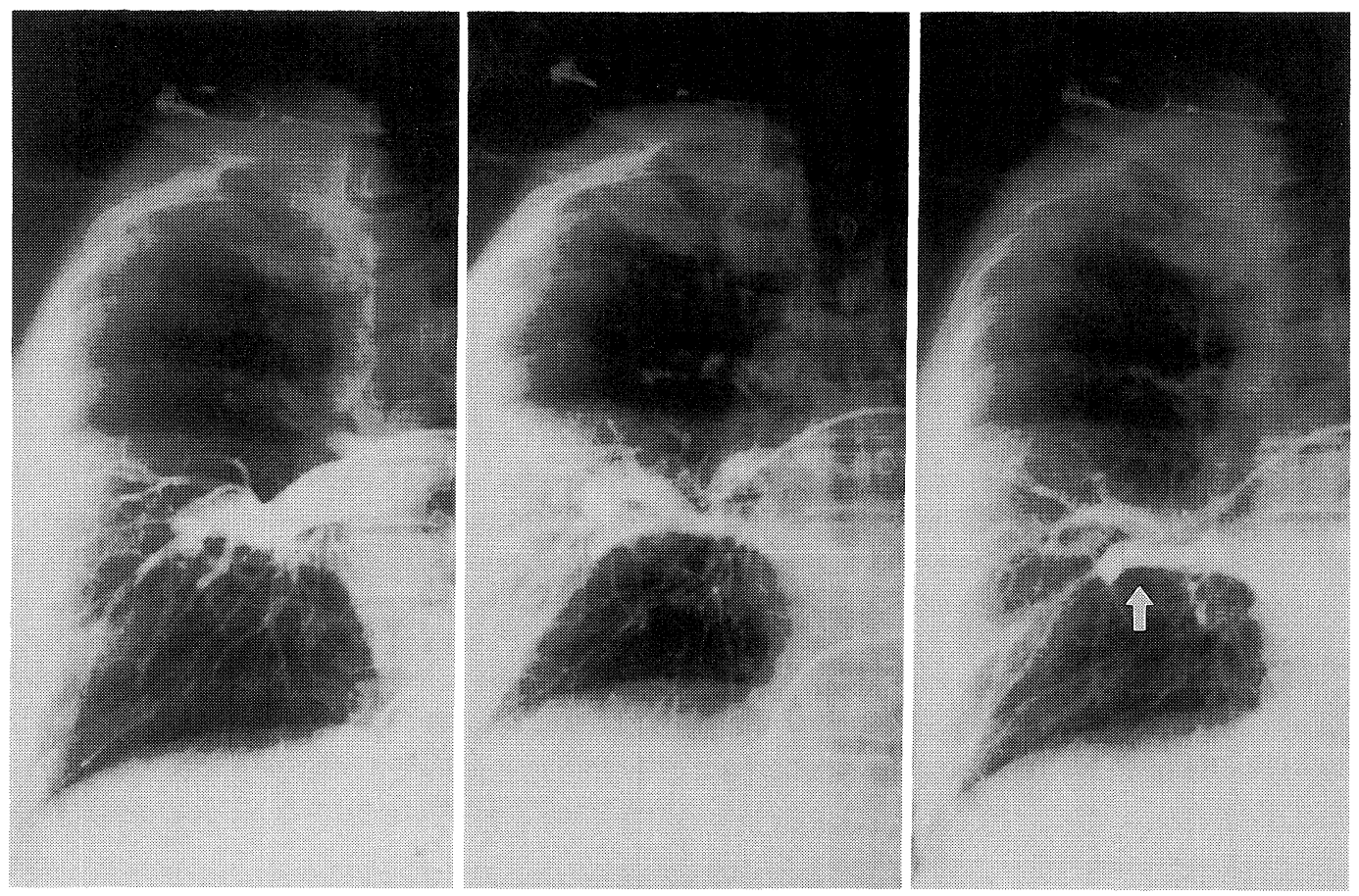

Figure 3. Selective right pulmonary angiogram (posteroanterior view). The pulmonary artery feeds the nodule and at the next phase the pulmonary vein is enhanced (arrow) when no other pulmonary vein is enhanced. 
graphy revealed that branches of the pulmonary artery to the bilateral inferior lobes were narrow and branched abnormally. The right superior, the right inferior, and the left superior lobes were hypovascular (Fig. 2). Selective right pulmonary angiography showed that the pulmonary artery fed the nodule, and at the next phase the pulmonary vein was enhanced when no other pulmonary veins were enhanced. Nevertheless, no obvious nidus was observed (Fig. 3). The shunt rate was calculated to be $17.4 \%$. The patient was diagnosed as having pulmonary arteriovenous fistula without hereditary hemorrhagic telangiectasia (Rendu-Osler-Weber disease). Neither surgery nor embolization therapy was performed, and the patient has been followed up carefully for 2.5 years on an outpatient basis without notable changes.

\section{Discussion}

Pulmonary arteriovenous fistula, a rare abnormality (2), is an abnormal connection between a pulmonary artery and a vein. The present case of pulmonary arteriovenous fistula was also associated with unequal branching of the pulmonary artery system. Because no concomitant occurrence of the two has been reported previously, a case such as this may be quite rare.

Although the classic clinical triad of dyspnea on effort, cyanosis, and clubbing has been reported, most patients are asymptomatic. In the present case, cyanosis and shortness of breath were observed, and could not be explained by pulmonary arteriovenous fistula alone when the small shunt rate was considered. One explanation was ventilation-perfusion mismatch due to unequal branching of the pulmonary artery system and poor pulmonary function due to chronic bronchitis and bronchiectasis.

The final diagnosis of pulmonary arteriovenous fistula was made based on the findings of pulmonary angiography. Anabtawi et al proposed a classification of pulmonary arteriovenous fistulas and aneurysms based on anatomic features (3) in which they described two groups of multiple small arteriovenous fistulas in addition to groups of large arteriovenous fistulas (i.e., multiple small arteriovenous fistulas without aneurysms and those with anomalous venous drainage). In the present case, no obvious nidus was observed on the pulmonary angiogram. One explanation may be that the nidus was not enhanced; another may be that there were multiple, small, undetected arteriovenous fistulas that drained into a pulmonary vein, similar to the multiple small arteriovenous fistulas described by Anabtawi and colleagues (3).

Surgery $(4,5)$ or embolization theapy $(6)$ is often prescribed in the case of serious complications such as rupture of the fistula and embolism. Treatment indications are the presence of symptoms, a large shunt rate, complications of the lesion, and progression (7). We did not choose surgical intervention in the present case because resection of the right middle lobe would have caused progression of the hypoxia in the already markedly hypovascular residual region. Embolization therapy was not performed because of the low shunt rate and concerns about rejection. However, embolization therapy must be planned if the symptoms or the nodular shadow progress and complications appear.

Pulmonary arteriovenous fistula is either congenital or acquired, but it is most frequently congenital. Secondary pulmonary arteriovenous fistula occurs with trauma, schistosomiasis, chronic hepatic cirrhosis, metastatic carcinoma, and actinomycosis (2). About $60 \%$ of congenital pulmonary arteriovenous fistulas occur with Rendu-Osler-Weber disease (8). The present case was considered to be congenital because there were no concomitant pathologies to cause secondary pulmonary arteriovenous fistula. In addition, this case was not associated with Rendu-Osler-Weber disease. Abnormalities of the bronchial system may occur with abnormalities of the pulmonary vascular system. However, in this case, no abnormality was considered to be present at least in the central part of the bronchial system based on the bronchoscopy findings.

There are several theories for the origin of congenital pulmonary arteriovenous fistulas. One is incomplete degeneration of the vascular septa, which divides the arterial and venous plexuses in the 2 nd month of gestation (3). Another theory proposes a defect in the terminal capillary loops, which allows dilatation and formation of a thin-walled vascular sac $(2,9)$. Unequal branching of the pulmonary artery system is believed to occur when the pulmonary vascular system is divided from the systemic vascular system, just before the pulmonary arteriovenous fistula is thought to be formed. These abnormalities are thought to have occurred in the early gestational stage although the etiology is unknown. It is possible that such a concomitant occurrence is not rare, and that many cases have simply not been identified. Careful examination of similar cases may clarify the etiology of pulmonary arteriovenous fistula. Therefore, the concomitant occurrence of unequal branching of the pulmonary artery system should be taken into consideration when found in patients with pulmonary arteriovenous fistula. Sluiter-Eringa et al reported that pulmonary angiography should be performed not only to confirm the diagnosis but also to identify new and unsuspected pulmonary arteriovenous fistulas (7). Furthermore, because it would have been impossible to diagnose the unequal branching of the pulmonary artery system without pulmonary angiography in the present case, this angiographic procedure should be included in the diagnostic work-up.

\section{References}

1) Churton T. Multiple aneurysms of pulmonary artery. Br Med J 1: 1223, 1897.

2) Prager RL, Laws KH, Bender HW. Arteriovenous fistula of the lung. Ann Thorac Surg 36: 231, 1983.

3) Anabtawi IN, Ellison RG, Ellison LT. Pulmonary arteriovenous aneurysms and fistulas. Ann Thorac Surg 1: 277, 1965.

4) Hepburn J, Dauphinee JA. Successful removal of hemangioma of the lung followed by the disappearance of polycythemia. Am J Med Sci 204: 681, 1942.

5) Dines DE, Arms RA, Bernatz PE, Gomes MR. Pulmonary arteriovenous fistulas. Mayo Clin Proc 49: 460, 1974.

6) Taylor BG, Cockerill EM, Manfredi F, Klatte EC. Therapeutic embolization of the pulmonary artery in pulmonary arteriovenous fistulae. Am J Med 64: 360, 1978. 


\section{Morimoto et al}

7) Sluiter-Eringa H, Orie NGM, Sluiter HJ. Pulmonary arteriovenous fistula. Diagnosis and prognosis in noncompliant patients. Am Rev Respir Dis 100: $177,1969$.

8) Boscher LH, Blake DA, Byrd BR. An analysis of the pathologic anatomy of pulmonary arteriovenous aneurysms with particular reference to the applicability of local excision. Surgery 45: 91, 1959.

9) Hodgson CH, Burchell HB, Good CA, Clagett OT. Hereditary hemorrhagic telangiectasia and pulmonary arteriovenous fistula. N Engl J Med 261: 625,1959 